\title{
BMJ Open Role of occupational therapy in musicians' health: a scoping review protocol
}

\author{
Bethany Villas, ${ }^{1}$ Uira Duarte Wisnesky, ${ }^{2}$ Sandra Campbell, ${ }^{3}$ Lauren Slavik, ${ }^{1}$ \\ Amynah S. Mevawala, ${ }^{2}$ Melisa N. Handl, ${ }^{4}$ Christine Guptill (i) ${ }^{5}$
}

To cite: Villas B, Duarte Wisnesky U, Campbell S, et al. Role of occupational therapy in musicians' health: a scoping review protocol. BMJ Open 2020;10:e040922. doi:10.1136/ bmjopen-2020-040922

- Prepublication history and additional material for this paper is available online. To view these files, please visit the journal online (http://dx.doi.org/10. 1136/bmjopen-2020-040922).

Received 26 May 2020 Revised 25 November 2020 Accepted 02 December 2020

Check for updates

(C) Author(s) (or their employer(s)) 2020. Re-use permitted under CC BY-NC. No commercial re-use. See rights and permissions. Published by BMJ.

${ }^{1}$ Occupational Therapy, University of Alberta, Edmonton, Alberta, Canada

${ }^{2}$ Faculty of Nursing, University of Alberta, Edmonton, Alberta, Canada

${ }^{3}$ University of Alberta Libraries, University of Alberta, Edmonton, Alberta, Canada

${ }^{4}$ Faculty of Law, University of Ottawa, Ottawa, Ontario, Canada

${ }^{5}$ Faculty of Health Sciences

School of rehabilitation sciences, University of Ottawa, Ottawa, Ontario, Canada

Correspondence to

Dr Christine Guptill;

cguptill@uottawa.ca

\section{ABSTRACT}

Review question/objective The purpose of this proposed review is twofold: first, to understand the role of occupational therapy presented in the musicians' health literature; and second, to explore the potential for this role. Introduction The intense movement, awkward postures, concentration and emotional communication required of musicians can place them at increased risk of musicrelated health conditions, such as musculoskeletal disorders and performance anxiety. The development of music-related health conditions can be emotionally and financially devastating. The role of occupational therapy in musicians' health has been previously discussed; however, no rigorous reviews of the scholarly literature have been published. We will, therefore, undertake a scoping review with the following research questions: (1) what is known about the role of occupational therapy in instrumental musicians' health? and (2) what is the potential role of occupational therapy in musicians' health?

Methods and analysis A preliminary search of Medline, CINAHL, SCOPUS and Web of Science was previously undertaken by the first author to determine the extent of the research on this topic and to confirm that no other reviews have been conducted or are in progress. Study selection and analysis will follow the Joanna Briggs Institute and the Preferred Reporting Items for Systematic Reviews and Meta-Analyses extension for scoping reviews guidelines for conducting a scoping review.

Ethics and dissemination Formal ethics approval is not required at our institution for a review of published literature. The results of this review will be shared through peer-reviewed publications, conference presentations and traditional and social media.

\section{INTRODUCTION}

Instrumental musicians are recognised for their skill and dedication to their occupation. In the fields of occupational therapy and occupational science, occupations are defined as activities and tasks in which people engage that are given value or meaning by the person or culture. ${ }^{1}$ For musicians, this can include practising, playing, learning and performing. However, the intense movement, awkward postures, concentration and emotional communication required of musicians can

\section{Strengths and limitations of this study}

This scoping review protocol follows the Joanna Briggs Institute and Preferred Reporting Items for Systematic Reviews and Meta-Analyses extension for scoping reviews guidelines for conducting scoping reviews.

- This is a novel scoping review that will provide a comprehensive review of what is known about the current and potential role of occupational therapy in the care of instrumental musicians' playing-related health conditions, adding to the potential care available for this vulnerable population.

- Papers of English and non-English language will be considered in selection to reduce language bias.

- This review is limited to evidence from peerreviewed, published papers, which may result in publication bias.

place them at increased risk of music-related health conditions, including musculoskeletal disorders such as repetitive strain disorders and mental health concerns such as performance anxiety. ${ }^{2}$ Because musicians' identities are often closely tied to their occupation, ${ }^{3}$ the development of music-related health conditions can be emotionally devastating. In addition, many musicians are self-employed, have very low incomes and lack access to workers' compensation and employer-paid health insurance schemes. ${ }^{45}$ As a result, a holistic approach to healthcare that considers a wide range of contextual factors (eg, social insurance schemes, relations with colleagues) and overlapping roles (eg, worker, teacher, musician) has been recommended for this vulnerable population. ${ }^{6}$

The role of occupational therapy in musicians' health has been previously discussed by Guptill $^{7}$ and Goodman and Staz ${ }^{8}$; however, to our knowledge, no peer-reviewed, rigorous reviews exist on this topic. Two research questions will direct this scoping review: (1) What is known about the role of occupational therapy in instrumental musicians' health and 
(2) What is the potential role of occupational therapy in musicians' health? This scoping review will help musicians and health practitioners understand the potential benefits of occupational therapy to enable meaningful occupation for those with music-related health conditions.

\section{METHODS}

\section{Study design}

To answer the research questions, a scoping review will be used. Scoping reviews are used to understand broad questions by collecting available literature related to a question. ${ }^{9}$ A scoping review was chosen to understand the role of occupational therapy in the current literature on musicians' health and to explore future areas where an occupational therapist may be beneficial.

To guide the review and synthesis process, Arksey and O'Malley's ${ }^{10}$ scoping review framework, as adapted by Levac et $a l,{ }^{9}$ will be followed. The review will proceed as follows: (1) identify the research question (2) identify the relevant studies (3) study selection (4) charting the data and (5) collating, summarising, and reporting results. We will not engage in the optional stage 6-consultation with the community-in this current study, although such consultation may form a part of future knowledge translation. The study will follow the Joanna Briggs Institute (JBI) and the Preferred Reporting Items for Systematic Reviews and Meta-Analyses (PRISMA) extension for scoping reviews. ${ }^{11} 12$ This protocol has been registered with the JBI Systematic Review Register.

\section{Step 1: identify the research question}

Two research questions, identified above, will direct our review, with the goal of understanding the role of occupational therapy in musicians' health.

\section{Step 2: search strategy}

The search strategy was developed with the collaboration of a librarian/expert searcher and then reviewed by a second librarian/expert searcher. A preliminary search of Medline, Cumulative Index to Nursing and Allied Health (CINAHL), SCOPUS, and Web of Science was undertaken to determine the extent of the research on this topic and to confirm that no other reviews have been conducted or are in progress. A search strategy including both text words and controlled vocabulary (eg, MeSH, EMTREE, etc) for the concepts 'musicians' and 'occupational therapy' will be conducted in the following databases: PROSPERO, Wiley Cochrane Library, Ovid Embase, Ovid Medline, Ovid PsycINFO, EBSCO CINAHL, EBSCO Répertoire International de Littérature Musicale, Web of Science and SCOPUS. PROSPERO was searched to ensure duplicate research is not already being conducted and to identify any other research groups currently working in the same area. Studies published since the inception year of each database will be considered for inclusion. The Medline search strategy (online supplemental appendix 1) will be adapted for each database by the third author, a health sciences librarian. In addition, the reference list of papers selected for full review will be searched to ensure the review is comprehensive. Results from the searches will then be imported into RefWorks (ProQuest, 2020) for management and selection.

\section{Step 3: study selection \\ Selection process}

Screening of abstracts will be conducted independently by four reviewers for assessment against the inclusion criteria. Two of the reviewers are occupational therapists, and one of these two will review each abstract. Each abstract will be screened in duplicate. Reviewers will screen each potentially useful abstract identified in the literature search by reading the title and abstract and applying the inclusion/exclusion criteria as reported below and then determining whether to include the study in the review. Every decision, with the reasons for inclusion/exclusion, will be recorded in the study screening spreadsheet. Once each reviewer has completed their screening process, the group will compare their results. Abstracts that are determined to be not relevant to the study will be excluded from the full-text review.

Full texts of remaining studies will be retrieved and independently assessed against the inclusion criteria by the four reviewers, and the process described for abstracts will be repeated. The results of the search, screening and selection will be reported in full, and presented in a PRISMA flow diagram in the final report as guided by Levac et al scoping review framework. ${ }^{9}$ During the abstract and full-text screening, any disagreements that arise between the reviewers will be resolved through discussion or with input from a third reviewer.

\section{Eligibility criteria}

Selection of the literature will be based on the following inclusion and exclusion criteria.

\section{Inclusion criteria}

All studies that focus, evaluate, comment on or explore the following will be included:

- Occupational therapy/therapist explicit involvement.

- Instrumental musician population.

- Health related.

\section{Exclusion criteria}

- Papers focusing solely on vocal musicians and/or non-instrumental musician performers.

- Interventions solely based on music therapy and not occupational therapy.

This scoping review will consider all settings in any geographic area/country. No date or language limit will be applied, enabling us to capture the full range of relevant studies for this review. No publication format limitations have been set; thus, database results may include different formats of publication to be considered (eg, abstracts). We will include primary research studies, including quantitative, qualitative and mixed-method study designs, and secondary research studies, including 
all forms of literature reviews. Grey literature will not be included in this review as the focus of this study is on published, peer-reviewed literature, which would guide and be the basis of education and clinical reasoning as occupational therapists.

\section{Data management}

All results from the database and hand searches will be imported into RefWorks (ProQuest, 2020). Duplicates and publications that do not directly relate to the research question will be excluded. The remaining citations will be imported into a spreadsheet for screening. Data will be extracted from the included studies using a structured data extraction form based on the research questions (online supplemental appendix 2).

\section{Step 4: data collection}

Data will be extracted from the included studies using a proposed structured data collection form, based on the research questions (online supplemental appendix 2). Information pertinent to the review questions will be recorded in the data extraction form. An iterative thematic analysis process and the Canadian Model of Occupational Performance and Engagement ${ }^{14}$ will be applied to each document so that themes related to the research question and the scope of practice of occupational therapy emerge. Charting the results will also be iterative, such that the data collection form may be calibrated to reflect new information and decisions by the reviewers. ${ }^{910}$

\section{Study selection}

The review team will trial the data collection form with five studies to ensure consistency, to gain familiarity with the search results, and to ensure that all relevant results are extracted. If modifications occur, they will be detailed in the final report. Authors of papers will be contacted to request missing or additional data, where required.

\section{Data items}

The variables currently identified for data collection are publication type, health-related content, participant type, healthcare providers involved and whether the article focuses on music therapy (as an exclusion data item).

\section{Outcomes and prioritisation}

The outcomes of interest will be the current and potential role of occupational therapy in instrumental musicians' health.

\section{Step 5: data synthesis}

The extracted data will be presented as a summary of the retrieved data in tabular form, categorised as follows: (1) authors and year of publication, (2) professional designation, (3) study purpose, (4) musician population, (5) type of study/methods, (6) treatment provided, (7) key points and (8) suggested occupational therapy role and scopes. Finally, the results will be presented in a narrative description that aligns with the research questions of this scoping review, which are to explore what is known about the current and potential role of occupational therapy in instrumental musicians' health.

\section{Patient and public involvement}

No patient or public involvement was sought for this scoping review protocol.

\section{LIMITATIONS}

A limitation of this scoping review is that the databases we will be searching contain only bibliographic information and abstracts, rather than full texts of articles, so articles containing only in-text references to our search terms cannot be retrieved. As such, studies relevant to our review may be missed. To decrease this limitation, the reference lists of, and citations in, the selected studies will be screened for relevance to our study.

\section{ETHICS AND DISSEMINATION}

Formal ethics approval is not required at our institution for a review of published literature. The results of this review will be shared through peer-reviewed publications, conference presentations and traditional and social media.

\section{Twitter Christine Guptill @healthmusicperf}

Acknowledgements Thank you to the support of CG, UDW, AM and SC for providing guidance and expertise in this process.

Contributors CG is the corresponding author, and conceived of the idea for this project. BV developed the research question and conducted a preliminary review to determine the feasibility of the research questions. BV, LS, UDW and CG participated in the development of the draft data extraction form. BV, CG, SC and UDW wrote the initial manuscript. UDW, AM and MH managed the process of manuscript revisions. All authors read and provided feedback on the revisions and final manuscript.

Funding The authors have not declared a specific grant for this research from any funding agency in the public, commercial or not-for-profit sectors.

Competing interests None declared.

Patient consent for publication Not required.

Provenance and peer review Not commissioned; externally peer reviewed.

Supplemental material This content has been supplied by the author(s). It has not been vetted by BMJ Publishing Group Limited (BMJ) and may not have been peer-reviewed. Any opinions or recommendations discussed are solely those of the author(s) and are not endorsed by BMJ. BMJ disclaims all liability and responsibility arising from any reliance placed on the content. Where the content includes any translated material, BMJ does not warrant the accuracy and reliability of the translations (including but not limited to local regulations, clinical guidelines, terminology, drug names and drug dosages), and is not responsible for any error and/or omissions arising from translation and adaptation or otherwise.

Open access This is an open access article distributed in accordance with the Creative Commons Attribution Non Commercial (CC BY-NC 4.0) license, which permits others to distribute, remix, adapt, build upon this work non-commercially, and license their derivative works on different terms, provided the original work is properly cited, appropriate credit is given, any changes made indicated, and the use is non-commercial. See: http://creativecommons.org/licenses/by-nc/4.0/.

ORCID iD

Christine Guptill http://orcid.org/0000-0002-5080-2672

\section{REFERENCES}

1 Townsend E. Enabling occupation: an occupational therapy perspective. Ottawa, ON: CAOT Publications ACE, 2002. 
2 Hagglund KL, Jacobs K. Physical and mental practices of music students as they relate to the occurrence of music-related injuries. Work 1996;6:11-24.

3 Guptill CA. The lived experience of professional musicians with playing-related injuries: a phenomenological inquiry. Med Probl Perform Art 2011;26:84-95.

4 Statistics Canada. Labour force survey, 2019. Available: http:// www23.statcan.gc.ca/imdb/p2SV.pl?Function=getSurvey\&SDDS= $3701 \&$ lang $=\mathrm{fr} \& \mathrm{db}=\mathrm{imdb} \& \mathrm{adm}=8 \& \mathrm{dis}=2$ [Accessed 24 May 2020].

5 Hill Strategies Research Inc. Situation of artists, 2019. Available: https://hillstrategies.com/wp-content/uploads/2019/07/arm_vol18_ no3.pdf [Accessed 24 May 2020].

6 Guptill CA. Survivors on the edge: the lived-experience of professional musicians with playing-related injuries (doctoral dissertation, 2010. Available: https://ir.lib.uwo.ca/cgi/viewcontent. cgi ?article $=7033 \&$ context $=$ digitizedtheses $[$ Accessed 24 May 2020].

7 Guptill C. Musicians' health: a developing role for occupational therapists. Occupational Therapy Now. , 2014: 16, 29-31. https:// www.caot.ca/document/3979/nov_AE_NOV_2014.pdf
8 Goodman G, Staz S. Occupational therapy for musicians with upper extremity overuse syndrome: patient perceptions regarding effectiveness of treatment. Med Probl Perform Art 1989;4:9-14.

9 Levac D, Colquhoun H, O'Brien KK. Scoping studies: advancing the methodology. Implement Sci 2010;5:69.

10 Arksey H, O'Malley L. Scoping studies: towards a methodological framework. Int J Soc Res Methodol 2005;8:19-32.

11 Aromataris E, Munn Z. Joanna Briggs Institute Reviewer's Manual. The Joanna Briggs Institute, 2017. Available: https:// reviewersmanual.joannabriggs.org/ [Accessed 24 May 2020].

12 Tricco AC, Lillie E, Zarin W, et al. PRISMA extension for scoping reviews (PRISMA-ScR): checklist and explanation. Ann Intern Med 2018;169:467-73 https://doi.org/

13 Moher D, Liberati A, Tetzlaff J, et al. Preferred reporting items for systematic reviews and meta-analyses: the PRISMA statement. BMJ 2009;339:b2535:332-6.

14 Townsend EA, Polatajko HJ. Enabling Occupation II: Advancing an Occupational Therapy Vision for Health, Well-being, \& Justice through Occupation. $2^{\text {nd }}$ Ed. Ottawa, ON: CAOT Publications ACE, 2013. 